\title{
BMJ Open Systematic review protocol for assessing central auditory functions of Alzheimer's disease and its preclinical stages
}

\author{
Dona M P Jayakody (D , ,,2 Hadeel Y Tarawneh (D) , ${ }^{1,2,3}$ Holly K Menegola, ${ }^{1,2}$ \\ Jessica M Yiannos, ${ }^{1,2}$ Peter L Friedland, ${ }^{1,4,5}$ Wayne J Wilson, ${ }^{6}$ Ralph N Martins, ${ }^{7,8}$ \\ Hamid R Sohrabi ${ }^{7,8,9}$
}

To cite: Jayakody DMP, Tarawneh HY, Menegola HK, et al. Systematic review protocol for assessing central auditory functions of Alzheimer's disease and its preclinical stages. BMJ Open 2020;10:e033342. doi:10.1136/ bmjopen-2019-033342

- Prepublication history for this paper is available online. To view these files, please visit the journal online (http://dx.doi. org/10.1136/bmjopen-2019033342).

Received 02 August 2019 Revised 06 May 2020 Accepted 12 May 2020

\section{Check for updates}

(c) Author(s) (or their employer(s)) 2020. Re-use permitted under CC BY-NC. No commercial re-use. See rights and permissions. Published by BMJ.

For numbered affiliations see end of article.

Correspondence to Hadeel Y Tarawneh; hadeel.tarawneh@research. uwa.edu.au and Dr Dona M P Jayakody; dona.jayakody@earscience. org.au

\section{ABSTRACT}

Introduction A number of studies have reported an association between peripheral hearing impairment, central auditory processing and Alzheimer's disease (AD) and its preclinical stages. Both peripheral hearing impairment and central auditory processing disorders are observed many years prior to the clinical manifestation of $A D$ symptoms, hence, providing a long window of opportunity to investigate potential interventions against neurodegenerative processes. This paper outlines the protocol for a systematic review of studies examining the central auditory processing functions in $A D$ and its preclinical stages, investigated through behavioural (clinical assessments that require active participation) central auditory processing tests.

Methods and analysis We will use the keywords and Medical Subject Heading terms to search the following electronic databases: MEDLINE, PsychINF0, PubMed, Scopus, EMBASE and CINAHL Plus. Studies including assessments of central auditory function in adults diagnosed with dementia, $A D$ and its preclinical stages that were published before 8 May 2019 will be reviewed. This review protocol will be reported according to the Preferred Reporting Items for Systematic Reviews and Meta-Analyses Protocols guidelines. Data analysis and search results will be reported in the full review. This manuscript has designed the protocols for a systematic review that will identify the behavioural clinical central auditory processing measures that are sensitive to the changes in auditory function in adults with $A D$ and its preclinical stages. Such assessments may subsequently help to design studies to examine the potential impact of hearing and communication rehabilitation of individuals at risk of $A D$.

Ethics and dissemination Ethical approval is not required as this manuscript only reports the protocols for conducting a systematic review as primary data will only be reviewed and not be collected. The results of this systematic review will be disseminated through publication and in scientific conferences.

PROSPERO registration number CRD42017078272.

\section{INTRODUCTION}

Globally, about 44 million people were living with Alzheimer's disease (AD) or related
Strengths and limitations of this study

- The systematic review, this protocol outlines, will contribute to strengthen the understanding of the strengths and limitations of behavioural central auditory function assessments in individuals with Alzheimer's disease (AD) and those at risk of developing AD.

- This systematic review protocol is described in accordance with the Preferred Reporting Items for Systematic Reviews and Meta-Analyses Protocols guidelines.

- There are no language restrictions imposed for the full-text literature search for this systematic review.

- Exclusion of articles with abstracts not in English can potentially mean that important findings are missed.

- The manuscript only describes a protocol for a systematic review. A follow-up manuscript will explore the systematic review results and meta-analysis of the data.

dementia in 2017 and this number is projected to reach 76 million by $2050 .{ }^{1}$ The preclinical and prodromal stages of $\mathrm{AD}$ include subjective cognitive decline (SCD), mild cognitive impairment (MCI) and dementia. ${ }^{2-4}$ Not all those with SCD or MCI may progress to $\mathrm{AD}$, only a certain per cent of individuals will proceed to the next neurodegenerative stage. ${ }^{5}$ The neuropathological changes associated with $\mathrm{AD}$ have been shown to start up to 20 years prior to the clinically manifested symptoms..$^{6}$ This long pre-clinical phase provides a rare opportunity for early detection and prevention of the disease, and it is now estimated that up to $35 \%$ of $\mathrm{AD}$ diagnoses worldwide could be delayed or prevented with early intervention and by making lifestyle changes whichthat reduce the identified modifiable risk factors for developing $\mathrm{AD} .{ }^{78}$ Identifying these early markers of $\mathrm{AD}$ as well as the potential factors which will help prevent or delay 
both age-related and pathological cognitive declines in $\mathrm{AD}$ becomes particularly important considering there is no cure or effective treatment currently insight.

According to Lancet International Commission on Dementia Prevention, Intervention, and Care report, mid-life hearing loss could account for $9 \%$ of the population attributable fraction of modifiable risk factors associated with increased risk of $\mathrm{AD} .^{7}$ The auditory system is comprised of two key components, the peripheral hearing system (outer, middle and inner ear and auditory nerve) and the central auditory pathway (cochlear nucleus to the auditory cortex), which are respectively involved in the detection and processing of incoming auditory signal. ${ }^{9}$ The central auditory system is responsible for key communication skills such as feature extraction and detecting small gaps in speech, which is crucial in speech discrimination and integrating and separating binaural auditory information. ${ }^{10}{ }^{11} \mathrm{~A}$ combination of these central auditory processing (CAP) skills facilitates the understanding of speech in background noise and spatial processing skills as well as language and information processing. ${ }^{1011}$

Results from longitudinal studies suggest that CAP skills, in the absence of a severe peripheral hearing loss, are associated with high incidences of cognitive decline and AD dementia. ${ }^{12-14}$ These studies have demonstrated that those with severe CAP disorders $(<50 \%$ scores on Dichotic Sentence Identification task) were at a greater risk for incident dementia with hazard ratios of $9.9(95 \%$ CI 3.6 to 26.7$){ }^{13}$

Further, a number of studies have reported impaired CAP skills in individuals with MCI. ${ }^{131516}$ The exact mechanism underlying the impaired CAP skills and cognitive decline is yet to be elucidated, two theories have been proposed to explain this association. The first theory suggests that it could result from independent decline in both CAP skills and cognition, exhausting cognitive reserve and exacerbating the deficits experienced in both CAP skills and cognitive abilities, manifesting as language, learning and communication difficulties. ${ }^{11}$ The second theory suggests that cortical degeneration of common neuronal structures, resulting from neurodegenerative disorders such as dementia and $\mathrm{AD}$, could lead to deficits in speech, hearing and cognitive processes. ${ }^{12} 17$

Numerous studies to date have concluded that there is a strong link between poor central auditory functions and the preclinical stages of AD. This link could provide a unique opportunity to easily identify people at high risk of developing, or who are in the preclinical phases of $\mathrm{AD}$, years before a formal diagnosis can be made. ${ }^{1819}$ Based on existing meta-analyses data, Norton $e t a l^{8}$ calculated the population attributable risk of $\mathrm{AD}$ worldwide, UK, USA and Europe for seven modifiable risk factors that have been associated with AD: diabetes, midlife hypertension, midlife obesity, physical inactivity, depression, smoking and low educational attainment. Based on the assumption of a causal relationship between $\mathrm{AD}$ and risk factors and timely intervention, the effect of risk factor reductions on $\mathrm{AD}$ projections from 2010 to 2050 for each of the seven risk factors was calculated. Results suggested that if the risk factor was reduced by $10 \%$ or $20 \%$ per decade for each of the seven risk factors, the projected cases of worldwide $\mathrm{AD}$ could be reduced by 8.3 to 16.2 million by $2050 .{ }^{8}$ It is important that we evaluate the validity and reliability of the central auditory function tests used in preclinical $\mathrm{AD}$ (SCD, MCI and dementia) before proceeding to develop tests or intervention strategies for people identified to have poor CAP skills.

This paper outlines the protocol that will be used to systematically review the behavioural central auditory function assessments of 40-85-year-old people diagnosed with $\mathrm{AD}$ and its preclinical stages and compares the results with the 40-85-year-old healthy adults with no cognitive impairment or memory complaints. The systematic review will also examine the (1) challenges pertaining to selection and administration of behavioural CAP assessments to those with impaired cognitive and/or daily living functions, (2) modifications to the standard central hearing assessment procedures to accommodate impaired cognitive and/or daily living functions and (3) the factors that influence the interpretation of test results. Two research questions will be investigated: (1) What are the most commonly utilised behavioural CAP tests for older adults diagnosed with $\mathrm{AD}$ or in preclinical stages and (2) What are the limitations of these measures and how can they be improved?

\section{METHODS AND ANALYSIS}

This protocol will be reported in accordance with the Preferred Reporting Items for Systematic Reviews and Meta-Analyses Protocols 2015 checklist. ${ }^{20}$

\section{Patient and public involvement}

This was a protocol paper for reviewing previously published papers. Therefore, no patient or patientrelated data were required, assessed or involved in any part of this protocol preparation.

\section{Criteria}

All papers related to behavioural central auditory functions and $\mathrm{AD}$ dementia, MCI and SCD that were published and available on searched databases before 8 May 2019 will be considered. The inclusion and exclusion criteria are described below.

\section{Population}

Adults aged between 40 and 85 years with typical 'amnestic MCI, dementia due to AD, non-amnestic MCI and SCD' will be included. Other cognitive impairments not related to $\mathrm{AD}$, including logopenic progressive aphasia, primary progressive aphasia, posterior cortical atrophy and frontal variant $\mathrm{AD}$, will be excluded. Participants with visual impairment also will be excluded.

\section{Intervention}

Behavioural central auditory function tests that measure (1) monaural low redundancy speech tests (low-pass 
filtered speech tests, speech-in-noise tests, speech-inmessage competition tests, time-compressed speech tests), (2) temporal processing (temporal ordering or sequencing, temporal resolution or discrimination, temporal integration or summation, temporal masking), (3) binaural interaction (masking-level difference, interaural timing and lateralisation/localisation, binaural fusion, rapidly alternating speech perception) and (4) dichotic listening (dichotic digits, dichotic sentences, staggered spondaic word test, dichotic consonant-vowels, dichotic rhymes) will be included. Auditory electrophysiological assessments will be excluded from the review.

\section{Comparator}

The control group will consist of healthy adults aged from 40 to 85 years. Those with cognitive complaints, neurocognitive impairment, impaired daily living functions and visual impairment will be excluded.

\section{Outcomes}

Only quantitative measurements will be included. Both cross-sectional and longitudinal studies that documented the changes of the central auditory functions of adults diagnosed $\mathrm{AD}$ and its preclinical stages will be included.

\section{Study type}

Randomised controlled trials, feasibility studies, pilot studies and clinical trials will be considered.

No language restrictions will be imposed during the literature search, however, the abstract should be available in
English. All studies should be original research published in a peer-reviewed journal.

\section{Information sources}

The following databases will be selected: MEDLINE, PsychINFO, PubMed, Scopus, EMBASE and CINAHL Plus electronic databases, which are the most likely to index reports of studies in audiology, speech and hearing, psychology, cognition, neuroscience, neuropsychology and cognitive sciences, psychiatry and mental health. A hand-search of references of the included articles will also be performed as well. Grey literature will be sought using Google Scholar, Open Grey, ClinicalTrials.gov and International Clinical Trials Registry Platform. Unpublished studies from conference proceedings, scientific meetings or thesis were included in the search to mitigate publication bias.

\section{Search strategy}

To maximise the sensitivity of the search strategy, the following will be used: MeSH terms in exploded mode (MEDLINE, PsychINFO and EMBASE), text searches or keywords (PubMed, CINAHL Plus and Scopus), with truncations, synonyms and different spellings. The searches will be conducted in the title, abstract and full-text fields. Each combination of identifiers between domains will be searched when occurring concurrently in the same report (refer to table 1).

Table 1 Keywords and Medical Subject Headings (MeSH) terms (explode) applied per domain in search databases

\begin{tabular}{|c|c|c|}
\hline Domain & Keywords & MeSH terms \\
\hline
\end{tabular}

Alzheimer's Alzheimer* disease OR Alzheimer's* OR Dementia OR AD OR cognitive impairment OR disease cognitive decline OR cognitive processing OR cognitive ability dementia OR Alzheimer ${ }^{\star}$ type dementia OR mild cognitive impairment OR MCI OR amnestic MCI OR amnestic mild cognitive impairment OR minimal cognitive impairment OR moderate cognitive

Dementia OR

Cognitive Dysfunction OR Cognitive defect impairment OR severe cognitive impairment OR memory complainers OR SMC OR subjective cognitive decline OR SCD, or cognitive impairment no dementia OR CIND 
In order to account for all the behavioural central auditory hearing assessments that were not covered by the keywords and MeSH terms, we will also include any test that measures monaural low redundancy speech/tones, dichotic listening tests consist of musical notes/chords, temporal processing and binaural interaction tasks.

A dual independent review will be applied to the search strategy as well as to the processes of identification and assessment of studies to reduce random errors and bias. ${ }^{21}$ The search strategy will also be reviewed by an independent librarian. ${ }^{22}$

\section{Data management}

All results from database and hand searches will be exported into EndNote V.X7 software (Thomson Reuters 2016) and then into the Covidence software program for systematic reviews (www.covidence.org). All the selected articles will then be independently screened using the Covidence software program.

\section{Study selection}

After removing the duplicates, titles and abstracts will be analysed against the study eligibility criteria (HYT and HM). Papers without abstract or abstracts that lack information will be kept for full-text analysis. The manuscripts kept for full-text analysis will be also screened by a third researcher (DMPJ). Discrepancies will be resolved by consensus. Reference lists and citations of the included papers and grey literature will be analysed to identify other potentially relevant papers (DMPJ).

\section{Data extraction}

The information extracted from screened articles will include the following (1) authors of the paper, year of publication and journal, (2) geographical location of the study, (3) demographics of the participants, (4) aims of the study, (5) types of the tests used, (6) outcomes measured, (7) major findings (results/conclusions), (8) limitations or difficulties. If data are not clearly presented in the research article, the authors of the original article will be contacted via email for further clarifications. Data will be reported under four subgroups: normal cognitive functions (controls), AD, MCI and SCD.

\section{Quality assessment}

The included studies will be assessed (internal validity or reliability) to address the following risk of bias: (1) selection bias, (2) performance bias, (3) detection bias, (4) attrition bias, (5) analysis bias and (6) reporting bias will be observed. A quality assessment tool developed by Thomas et al. ${ }^{23}$ will be used to systematically evaluate the methodological quality of the quantitative study designs. The findings will be analysed and a table of evidences will be constructed to grade the recommendations based on American Society of Plastic Surgeons' (ASPS) Evidence Rating Scale for Therapeutic Studies ${ }^{24}$ scale for grading recommendations.

\section{Meta-analytic approach}

The meta-analysis will be conducted using the Comprehensive Meta-Analysis (CMA V.3, Biostat, Englewood, USA). The synthesised effect size will be reported as the standard difference in mean with $95 \%$ CI. The standard mean difference indicates the difference between the means of two groups divided by their pooled SD. The data for the meta-analysis will be entered as continuous outcomes under the random effects model to account for variations between study methodologies. Heterogeneity will be assessed using the Q-value statistic and the $I^{2}$ statistic to indicate heterogeneity as a percentage. A $\mathrm{p}$ value $<0.05$ will be considered statistically significant for all analyses.

\section{DISCUSSION}

The main aim of this study is to systematically review the behavioural central auditory function assessments that have been used in studies with participants diagnosed with $\mathrm{AD}$ or in its preclinical stages. The results of the review will provide information about behavioural central auditory measures that are sensitive to the changes in auditory function in older adults with $\mathrm{AD}$ and its preclinical stages. This will help to design clinical trials to examine the potential impact of hearing and communication rehabilitation for individuals at risk of $\mathrm{AD}$. We postulate that this review will be useful to a variety of stakeholders who have an interest in the care of $\mathrm{AD}$ and related disorders.

\section{Author affiliations}

${ }^{1}$ Ear Sciences Centre- Faculty of Medical \& Health Sciences, University of Western Australia, Crawley, Western Australia, Australia

${ }^{2}$ Ear Science Institute Australia, Subiaco, Western Australia, Australia

${ }^{3}$ School of Human Sciences, University of Western Australia, Crawley, Western Australia, Australia

${ }^{4}$ Department of Otolaryngology Head Neck Skull Base Surgery, Sir Charles Gairdner Hospital, Nedlands, Western Australia, Australia

${ }^{5}$ School of Medicine, University of Notre Dame Australia, Fremantle, Western Australia, Australia

${ }^{6}$ School of Health and Rehabilitation Sciences, The University of Queensland, St Lucia, Queensland, Australia

${ }^{7}$ School of Medical and Health Sciences, Edith Cowan University, Joondalup, Western Australia, Australia

${ }^{8}$ Department of Biomedical Sciences, Faculty of Medicine and Health Sciences, Macquarie University, Sydney, New South Wales, Australia

${ }^{9}$ College of Science, Health, Engineering and Education, Murdoch University, Murdoch, Western Australia, Australia

\section{Twitter Dona M P Jayakody @dona.jayakody}

Contributors DMPJ, PLF and HRS contributed to the development of the idea and protocols; HYT provided input into the meta-analysis; WJW, HKM, RNM and JMY reviewed the protocols and provided input for revisions.

Funding The authors have not declared a specific grant for this research from any funding agency in the public, commercial or not-for-profit sectors.

Competing interests None declared.

Patient consent for publication Not required.

Provenance and peer review Not commissioned; externally peer reviewed.

Open access This is an open access article distributed in accordance with the Creative Commons Attribution Non Commercial (CC BY-NC 4.0) license, which 
permits others to distribute, remix, adapt, build upon this work non-commercially, and license their derivative works on different terms, provided the original work is properly cited, appropriate credit is given, any changes made indicated, and the use is non-commercial. See: http://creativecommons.org/licenses/by-nc/4.0/.

\section{ORCID iDs}

Dona M P Jayakody http://orcid.org/0000-0001-5814-4355

Hadeel Y Tarawneh http://orcid.org/0000-0003-1860-9167

\section{REFERENCES}

1 World Alzheimer Report. The global impact of dementia an analysis of prevalence, incidence, cost and trends, 2018. Available: https:// www.alz.co.uk/research/world-report-2018

2 Albert MS, DeKosky ST, Dickson D, et al. The Diagnosis of Mild Cognitive Impairment due to Alzheimer's Disease: Recommendations from the National Institute on Aging-Alzheimer's Association Workgroups on Diagnostic Guidelines for Alzheimer's Disease. Focus 2013;11:96-106.

3 McKhann GM, Knopman DS, Chertkow H, et al. The diagnosis of dementia due to Alzheimer's disease: recommendations from the National Institute on Aging-Alzheimer's association workgroups on diagnostic guidelines for Alzheimer's disease. Alzheimers Dement 2011;7:263-9.

4 Sohrabi HR, Weinborn M, Laske C, et al. Subjective memory complaints predict baseline but not future cognitive function over three years: results from the Western Australia memory study. Int Psychogeriatr 2019;31:513-25.

5 Sohrabi HRaW M. Cognitive Impairments in Alzheimer's Disease and Other Neurodegenerative Diseases. In: Martins RN, Brennan CS, Fernando WB, et al, eds. Neurodegeneration and Alzheimer's disease, 2019.

6 Villemagne VL, Burnham S, Bourgeat $P$, et al. Amyloid $\beta$ deposition, neurodegeneration, and cognitive decline in sporadic Alzheimer's disease: a prospective cohort study. Lancet Neurol 2013;12:357-67.

7 Livingston G, Sommerlad A, Orgeta V, et al. Dementia prevention, intervention, and care. The Lancet 2017;390:2673-734.

8 Norton S, Matthews FE, Barnes DE, et al. Potential for primary prevention of Alzheimer's disease: an analysis of population-based data. Lancet Neurol 2014;13:788-94.

9 Katz J. Handbook of clinical audiology. 7th edn. Philadelphia: Wolters Kluwer Health/Lippincott Williams \& Wilkins, 2015.

10 Fortunato S, Forli F, Guglielmi V, et al. A review of new insights on the association between hearing loss and cognitive decline in ageing. Acta Otorhinolaryngol Ital 2016;36:155-66.
11 Musiek FE, Chermak GD. Handbook of central auditory processing disorder volume 1 auditory neuroscience and diagnosis. Volume 1. 2nd edn. San Diego, USA: Plural Publishing, Inc, 2013.

12 Gates GA, Anderson ML, Feeney MP, et al. Central auditory dysfunction in older persons with memory impairment or Alzheimer dementia. Arch Otolaryngol Head Neck Surg 2008;134:771-7.

13 Gates GA, Anderson ML, McCurry SM, et al. Central auditory dysfunction as a harbinger of Alzheimer dementia. Arch Otolaryngol Head Neck Surg 2011;137:390-5.

14 Gates GA, Beiser A, Rees TS, et al. Central auditory dysfunction may precede the onset of clinical dementia in people with probable Alzheimer's disease. J Am Geriatr Soc 2002;50:482-8.

15 Edwards JD, Lister JJ, Elias MN, et al. Auditory processing of older adults with probable mild cognitive impairment. J Speech Lang Hear Res 2017;60:1427-35.

16 Idrizbegovic E, Hederstierna C, Dahlquist M, et al. Central auditory function in early Alzheimer's disease and in mild cognitive impairment. Age Ageing 2011;40:249-54.

17 Iliadou V, Kaprinis S, Iliadou V. Clinical psychoacoustics in Alzheimer's disease central auditory processing disorders and speech deterioration. Ann Gen Hosp Psychiatry 2003;2:12.

18 Jessen $\mathrm{F}$, Wolfsgruber $\mathrm{S}$, Wiese $\mathrm{B}$, et al. Ad dementia risk in late $\mathrm{MCl}$, in early $\mathrm{MCl}$, and in subjective memory impairment. Alzheimers Dement 2014;10:76-83

19 Rönnlund M, Sundström A, Adolfsson R, et al. Subjective memory impairment in older adults predicts future dementia independent of baseline memory performance: evidence from the Betula prospective cohort study. Alzheimers Dement 2015;11:1385-92.

20 Moher D, Shamseer L, Clarke M, et al. Preferred reporting items for systematic review and meta-analysis protocols (PRISMA-P) 2015 statement. Syst Rev 2015;4:1.

21 Eden J, Levit L, Berg A. Finding what works in health care standards for systematic reviews. Washington: National Academies Press, 2011.

22 Crowther M, Lim W, Crowther MA. Systematic review and metaanalysis methodology. Blood 2010;116:3140-6.

23 Thomas BH, Ciliska D, Dobbins $\mathrm{M}$, et al. A process for systematically reviewing the literature: providing the research evidence for public health nursing interventions. Worldviews Evid Based Nurs 2004;1:176-84.

24 American Society of Plastic Surgeons (ASPS). Evidence-based clinical practice guidelines. Retrieved January 10, 2016, 2011 Available: http://www.plasticsurgery.org/Documents/medicalprofessionals/health-policy/evidence-practice/ASPS-Rating-ScaleMarch-2011.pdf 